CORRECTION

https://doi.org/10.1038/s41586-019-1394-x

\title{
Author Correction: The bone marrow microenvironment at single-cell resolution
}

Anastasia N. Tikhonova, Igor Dolgalev, Hai Hu,

Kishor K. Sivaraj, Edlira Hoxha, Álvaro Cuesta-Domínguez,

Sandra Pinho, Ilseyar Akhmetzyanova, Jie Gao,

Matthew Witkowski, Maria Guillamot, Michael C. Gutkin,

Yutong Zhang, Christian Marier, Catherine Diefenbach,

Stavroula Kousteni, Adriana Heguy, Hua Zhong,

David R. Fooksman, Jason M. Butler, Aris Economides,

Paul S. Frenette, Ralf H. Adams, Rahul Satija,

Aristotelis Tsirigos \& Iannis Aifantis

Correction to: Nature https://doi.org/10.1038/s41586-019-1104-8, published online 10 April 2019.

In this Article, the citation to 'Fig. 5e' should have been 'Fig. $5 \mathrm{~d}$ ' following the text: “...average expression levels of the previously reported population-specific gene signatures ${ }^{45}$ ". In addition, the citation to 'Fig. 5f' should have been 'Fig. 5e' following the text: “...ectopically overexpressed across all HSPC subsets". The original Article has been corrected online. 